\title{
Cuando crear sinergia no siempre es Salud: Análisis y propuesta en la evolución del Sistema de Salud en Perú
}

\author{
When creating Synergy is not always Health: Analysis and Proposal on the Evolution of the Health System \\ in Peru
}

\author{
Luis Fernando Llanos Zavalaga 1,a,b, José Alberto Castro Quiroz ${ }^{1,2, a, c, d}$, Juan Ortiz Fernández ${ }^{1,2, a}$, Claudio \\ Willbert Ramírez Atencio a,d,e
}

\section{RESUMEN}

Los sistemas de salud, basados en los principios de atención primaria, entendida como primer contacto y atención continua, integral, integrada, y coordinada, logran mejores resultados y mayor equidad en salud que los sistemas con orientación centrada sólo en la atención especializada. La propuesta de Redes Integradas de Salud (RIS) en Perú señala que operarán mediante una cartera de atención integral de salud en redes, que atiendan a las necesidades de la población. El presente artículo de revisión reconoce que para ello se debe impulsar un proceso de modificación del sistema de salud, basado en la Atención Primaria, reconociendo las lecciones aprendidas, debe identificar los valores y principios que generen los cimientos para el desarrollo de políticas de estado en salud centradas en las prioridades nacionales de manera que los cambios sociales respondan a las necesidades y expectativas de toda la población. Ello debe impulsar cambios en la legislación, en criterios evaluativos, en generación y asignación de los recursos, enfoque prestacional y en la operación del sistema sanitario a través de sus componentes organizacionales y funcionales que permiten organizar las políticas, programas y servicios. El desarrollo de RIS en grandes ciudades requiere cambiar la lógica de la prestación materno infantil, hacia un enfoque integrado basado en el perfil epidemiológico, de riesgos y vulnerabilidades, teniendo como motor impulsor, un robusto sistema de referencia y contrarreferencia integrado (emergencias, ambulatorio, hospitalización). Se debe actualizar y aprobar un modelo de atención, remodelar la estructura, adecuar la actual infraestructura, infoestructura, recurso humano, equipamiento y los procesos operativos (operaciones) al interior de los establecimientos de salud certificados y adecuados de la RIS. PALABRAS CLAVE: Atención primaria de salud, sistemas nacionales de salud, colaboración intersectorial, organización y administración, Perú. (Fuente: DeCS BIREME).

\section{SUMMARY}

Health systems, based on primary care principles, understood as first contact and continuous, comprehensive, integrated, and coordinated healthcare, achieve better results and greater equity in health than systems with a focus only on specialized care. The proposal of Integrated Health Networks (RIS) in Peru indicates that they will operate through a portfolio of comprehensive healthcare in networks, which meet the needs of the population. This review article recognizes that for this, a process of modifying the health system based on Primary Care should be promoted, acknowledging the lessons learned, identifying the values and principles that generate the foundations

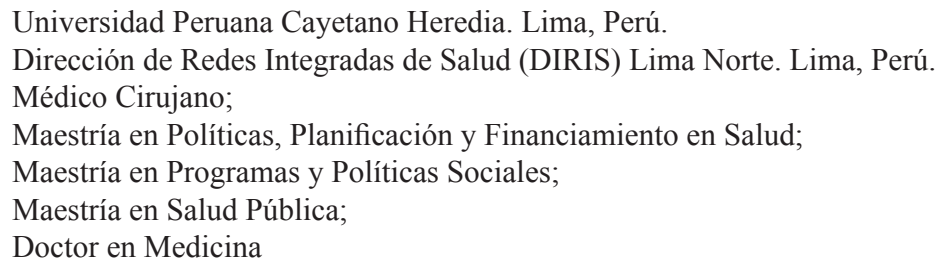


for the development of state health policies focused on national priorities so that social changes must respond to the needs and expectations of the population. This should promote changes in legislation, in evaluative criteria, in the generation and allocation of resources, in performance approach and in the operation of the health system through its organizational and functional components that allow an adequate organization of health policies, programs and services. The development of RIS in larger cities requires changing the focus on mother-child provision towards an integrated approach based on the epidemiological, risks and vulnerabilities profile, having as a driving engine, a robust integrated referral and counter-referral system (emergencies, outpatient, and hospitalization). A model of care must be updated and approved, modeling the structure, adapting the current infrastructure, human resources, equipment and operational processes (operations) within the certified and adequate health facilities of the RIS.

KEYWORDS: Primary health care, national health systems, intersectoral collaboration, organization and administration, Peru. (Source: MeSH NLM).

\section{INTRODUCCIÓN}

Se reconoce que los sistemas de salud, basados en los principios de la atención primaria, entendida como primer contacto, atención continua, integral e integrado, y coordinada, logran mejores resultados en salud y mayor equidad en salud que los sistemas con una orientación centrada sólo en la atención especializada. En diversos países se viene promoviendo el desarrollo de sistemas integrados de salud como una estrategia para mejorar el acceso, calidad y la continuidad de los servicios de una manera más eficiente, especialmente para las personas con necesidades complejas (por ejemplo, vulnerabilidades y morbilidades múltiples). Por otro lado, se sabe que un sistema segmentado y fragmentado da como resultado una atención subóptima, un mayor costo debido a la duplicación y una mala calidad de la atención, una realidad que no puede continuar; y para conocer los factores críticos que obstaculizan o facilitan la integración desde una perspectiva de atención primaria, se necesita un marco conceptual para hacer descripciones sistemáticas y comparables de estas iniciativas.

Lamentablemente, el concepto de atención o cuidado integrado puede ser ambiguo, y su uso varía en su alcance y valores subyacentes. Para este artículo usaremos la definición de cuidado integrado (del inglés, integrated care) como la búsqueda de conectar el sistema de salud (agudo, médico primario y calificado) con otros sistemas de servicios humanos (por ejemplo, servicios sociales, de atención a largo plazo, educación y servicios vocacionales y de vivienda) para mejorar los resultados de mediano y largo plazo (clínicos, satisfacción y eficiencia).

En el caso del Perú, la propuesta de Redes Integradas de Salud señala que ellas operan a través de una cartera de atención integral de salud en redes, que atienden a las necesidades de la población, en todos los establecimientos de salud del Ministerio de Salud y de los gobiernos regionales (1).

En este sentido, en este artículo se hará una revisión de la literatura sobre el desarrollo del Sistema de Salud en el Perú, enfatizando en la Atención Primaria en Salud hasta la tendencia en cuidado integral, para arribar a la propuesta de Redes y el abordaje organizacional que debe desarrollarse en las grandes ciudades, a fin de que las sinergias que se establecen en las Redes de Servicios de Salud, generen Salud.

\section{Evolución del sistema de salud en el Perú}

El sistema de salud (tabla 1) ha evolucionado presentando diversos periodos (1-8):

El desarrollo de la teoría salubrista lleva a reconocer que, para impulsar un proceso de modificación de un sistema de salud basado en la Atención Primaria de la salud (APS), se deben tener: "lecciones aprendidas y la identificación de valores esenciales para establecer las prioridades nacionales y para evaluar si los cambios sociales responden o no a las necesidades y expectativas de la población; principios que otorgan los cimientos para las políticas de salud, la legislación, los criterios evaluativos, la generación y asignación de los recursos y para la operación del sistema de salud; elementos que en un sistema de salud basados en la APS son los componentes organizacionales y funcionales que permiten organizar las políticas, los programas y los servicios (9).

\section{Propuesta de redes integradas de salud}

Se reconoce que la implementación y el desarrollo de las redes integradas de salud debe incluir los cuatro tipos de integración reconocidas: organizacional, 
Tabla 1. Evolución del Sistema de Salud, según periodos.

\begin{tabular}{|c|c|c|c|}
\hline Periodo & Años & Principales hechos & Observaciones \\
\hline Antecedentes & $1821-1922$ & $\begin{array}{l}\text { - Inicio de la atención en salud por parte del Estado } \\
\text { (1) } \\
\text { - 1903: Nace la Dirección General de Salubridad } \\
\text { (Ministerio de Fomento), con dos secciones: } \\
\text { Higiene y Demografía. } \\
\text { - 1922: Se crean las Oficinas Sanitarias } \\
\text { Departamentales como delegaciones }\end{array}$ & $\begin{array}{l}\text { 1933: Dr. Manuel Núñez Butrón, médico } \\
\text { sanitario departamental inicia su labor en } \\
\text { Puno, realizando un trabajo doctrinario a } \\
\text { nivel preventivo, promocional y curativo } \\
\text { que luego sería considerado pionero de } \\
\text { la Atención Primaria en Salud. }\end{array}$ \\
\hline
\end{tabular}

Inicios $\quad 1923-1960 \quad-$ 1935: El 5 de octubre se crea el Ministerio de

Organizativos

Atención

Primaria de

Salud

UDES, UTES y

1980- 1989

DIRESAS

$1961-1979$

Salud Pública, Trabajo y Previsión Social (Ley No8124). Secciones: salubridad, trabajo, previsión social y asuntos indígenas.

- 1942: El Ministerio se convierte en el Ministerio de Salud Pública y Asistencia Social (MSPAS).

1957: Se crean Áreas de Salud (D.S N¹05-

- SP-57). Territorios sanitarios como organismos "técnico- administrativos para promover, proteger u recuperar la salud de los habitantes del país" (2).

1968. Cambia el nombre Ministerial hacia Ministerio de Salud (MINSA).

- 1978: Declaración de Alma Ata. Punto VI: "La atención primaria forma parte integrante tanto del sistema nacional de salud, del que constituye la función central y el núcleo principal, como del desarrollo social y económico global de la comunidad". (3)

- Resolución XV (OPS/OMS): los SILOS son "política social en la que los aspectos relacionados con las estructuras de poder y las políticas de gobierno se entrelazan con factores culturales, de financiamiento y desarrollo económico, y de organización y administración del Estado en general y de los sistemas de salud". (4)

1978: Se crea el Sistema Nacional de Servicios

- de Salud (Decreto Ley $N^{\circ}$ 22365), con un rol supervisor y coordinador del MINSA en adición a su rol Normativo.

1979: Constitución Política del Perú, señala la

- responsabilidad del Poder Ejecutivo en organizar un sistema nacional de salud descentralizado y desconcentrado.

1985: Se crean las Unidades Departamentales de Salud (Decreto Legislativo Nº 351 - Ley orgánica Salubridad. del sector salud).

- 1987: Se aprueba la organización básica del Ministerio de Salud (Decreto Supremo N ${ }^{\circ}$ 02287-SA). Reglamento general de organización y funciones de UDES (R.M. $\mathrm{N}^{\circ}$ 160-89-SA/DM), determinó ámbito, estructura orgánica y funciones básicas en Unidades Territoriales de Salud, órganos responsables de la atención integral de salud de la población de una determinada área geográfica (Distrito o Provincia).
Las Áreas de Salud, fueron estructuradas por criterio geográfico, en unidades de salud, o dependencias ejecutivas. Son el antecedente inicial de las Regiones de Salud o DIRESAS.

APS, Estrategia para lograr la meta Salud para todos al año 2000, a través de los Sistemas Locales de Salud (SILOS); en el Perú, ZONADIS.

La salud centrada en la persona y basada en la población. Reconoce que los problemas de salud no son sinónimos de aspectos biológicos, o biomédicos (perspectiva biopsicosocial), que aborda las necesidades relacionadas con la salud en una población, incluye características políticas, sociales, económicas, culturales y ambientales, para el bienestar en una población.

- Durante la década de los 80 s, sigue lo establecido en Alma Ata.

Lineamientos de Política en Salud (MINSA 1982 - 1985), tuvieron como primer punto: Iniciar la elaboración del Proyecto de Ley que norme la Organización y funcionamiento de un Sistema Nacional Descentralizado y Desconcentrado de acuerdo al mandato de la constitución de 1979 y el funcionamiento del Sistema Nacional de Servicios de Salud en tanto se implante Dicha Ley. (5) 
Sistema

1990-1992

Nacional de

Salud

Invertir en

$1993-1999$

Salud - enfoque

materno infantil
- 1990: Nace el Sistema Nacional de Salud (Decreto Legislativo $\mathrm{N}^{\circ}$ 584). No fue oportunamente reglamentado. Su Consejo no fue conformado. (7)

- 1991: Lineamientos de Política en Salud MINSA 1991 se denominaron: "Hacia un Sistema Nacional Regionalizado e Integrado de la Salud".

Establecieron como Políticas: La Descentralización y regionalización en Salud, y la Recuperación de los EESS. (9)

- 1992: MINSA incluye las ZONADIS.

- 1992: Se crean DIRESAS (D.S. 002-92-SA). Órganos desconcentrados, encargados de ejecutar las normas emitidas por los órganos técnicoadministrativos del nivel central y de delimitación, organización e implementación de ZONADIS.

- 1992: Se crean Unidades Básicas de Servicios de Salud (R.M. N ${ }^{\circ}$ 0805-92-SA/DM). Órganos desconcentrados de las direcciones de salud en el ámbito correspondiente a ZONADIS, responsables de articular, apoyar, supervisar y evaluar los servicios públicos de salud y servir de apoyo técnico a la gestión del Consejo Zonal de Salud y sus EESS.

- 1993: El Banco Mundial publica Informe "Invirtiendo en Salud".

- 1993: Directiva norma procesos de reorganización y reestructuración de direcciones regionales y subregionales de salud de los gobiernos regionales (R.M. $\mathrm{N}^{\circ}$ 0020-93-SA/DM), especificándose la organización del sector salud en el nivel regional como: direcciones regionales y sub-regionales de salud, y ZONADIS.

- 1994: Se inicia la Focalización del Gasto Social con la implementación del Programa de Focalización del Gasto Social en Salud (PFGSS), que luego cambiaría de nombre a Programa Salud básica para Todos (PSBPT). Inician los Comités Locales de Administración Compartida (CLAS)

- 1996: Nace la propuesta internacional de Cuidado Integrado (integrated care).
MINSA en reglamento de organización y funciones: ZONADIS "como el nivel primario de la organización del Sistema Nacional de Salud, constituidas con el objetivo de brindar atención integral de salud en espacios geo-sociales previamente

definidos". Sin embargo, la experiencia de los SILOS no se consolidó en el Perú, quedando sólo la propuesta de organización del sistema de salud en base a criterios geográfico-poblacional con diversos nombres como Unidades Territoriales, Unidades Básicas de Servicios de Salud, ZONADIS.

Mensajes fundamentales del Informe son: Mejorar el gasto público en salud y Promover la diversidad y competencia. Reducir el gasto público en el tercer nivel, formación de especialistas e intervenciones con escasa mejora de salud. Se alienta los Seguros sociales o privados para ampliar cobertura de servicios esenciales.

El PSBPT, focalizó un paquete básico de atención para zonas de pobreza y pobreza extrema.

Lineamientos de Política de Salud, en la década de los 90s, establecidos en el Libro Azul, dejando de lado la Atención Primaria de Salud. (8).

- Según el Institute for Healthcare Improvement el cuidado integrado tiene como propósito contribuir a mejorar la salud de la población (cuidado), mejorar las experiencias individuales de atención (efectividad de atención) y reducir los costos de la atención per cápita (eficiencia), conocidos como el triple aim.(xx).

- Se expresa en cinco componentes: Enfoque en individuos y familias; Rediseño de servicios y estructuras de atención primaria; Gestión de la salud de la población; Plataforma de control de costos; e, Integración y ejecución de sistemas. 
Sistema

Nacional

Coordinado y

Descentralizado

de Salud

\section{APS renovada \\ y Redes}

Integradas de

Servicios de

Salud
$2000-2005$

$-200$ estableció como Objetivo 4: la reducción de la mortalidad infantil, como Objetivo 5: la mejora de la salud materna y como Objetivo 6: combatir el HIV/SIDA, malaria y otras, como tuberculosis. - 2001: Se crea el Seguro Integral de Salud sobre la base de los Seguros Escolar Gratuito (1997) y Materno Infantil (1998), enfatizándose en el Sistema de Salud el enfoque de cuidado de salud, priorizando la población materno infantil en la prestación de servicios de salud.

- 2002: Se crea el Sistema Nacional Coordinado y Descentralizado de Salud (SNCDS) (Ley N ${ }^{\circ}$ 27813).

- Normativamente en dicho año se da la Ley Orgánica de los Gobiernos Regionales que define las competencias y funciones del sector salud a ser transferidas desde el Gobierno Nacional (Ley orgánica de Gobiernos Regionales (Ley $\mathrm{N}^{\circ}$ 27867).

2005 - 2010 - La Organización Panamericana de la Salud (OPS) reimpulsa la APS renovada

- 2005: Se publica la Renovación de la APS en las Américas, abordando la fragmentación de los servicios de salud y las Redes Integradas de Servicios de Salud (RISS) como alternativa.

- 2008: Informe sobre Salud en el Mundo "La APS más necesaria que nunca", establece cuatro grandes reformas en APS. La segunda reforma aborda la Prestación de Servicios de Salud. (el modelo de atención, fragmentación y gestión).

- 2010: Se promulga la Ley 29344 (Ley de Aseguramiento Universal en Salud), que considera la Salud como derecho y no sólo como inversión

MAIS-BFC RIAPS/ RIS

- enfoque

integrado
2011- 2016

- 2011: Nace el MAIS-BFC (RM No 464-2011/ MINSA). El Modelo se basa en dos dimensiones: a) operativa: se debe desarrollar desde los establecimientos de salud en tres sujetos de intervención: persona, familia y comunidad; b) política: los determinantes sociales a intervenir a través de un trabajo multisectorial con participación de todos los niveles de gobierno.

- 2013: Marco normativo, para el funcionamiento de las redes integradas de atención primaria de salud (Decreto Legislativo $\mathrm{N}^{\circ} 1166$ ).

- 2018: Redes Integradas de Salud (Ley N 30885). Ámbito de intervención, establecimientos de salud del MINSA y Gobiernos Regionales, e integración opcional para los establecimientos de salud de los otros subsistemas. (1)

2019
Lineamientos de Política de Salud para el periodo 2002-2012 identificaron como prioridad de salud, la segmentación e irracionalidad en el sector salud y como su respuesta, la creación del SNCDS y el Modelo de Atención Integral en Salud - MAIS.(10) Se re-prioriza el enfoque en la atención del cuidado integral de la salud.

- Se emite el Lineamiento de Política: Promoción de la salud y prevención de la enfermedad, y crea la Dirección Ejecutiva de Promoción de Salud, para mejorar la deficiente salud ambiental, reducir la alta prevalencia de enfermedades. trasmisibles e incremento de no transmisibles, elevada desnutrición y mortalidad materno-infantil.

Lineamientos de Política Sectorial 2009-2010, establecen como Objetivo Sanitario la Salud Materno Infantil y la Desnutrición. Y como Estrategias para lograrlo al Aseguramiento Universal (AUS), la Descentralización y el Fortalecimiento del primer nivel de atención. (11)

- La Salud se asume como un Derecho Universal.

- Los Lineamientos de Política Sectorial 2011 - 2016 propiciaron el Fortalecimiento de la Rectoría en Salud a través de Diseñar, conducir, supervisar y regular el Sistema Nacional de Salud. En una clara alusión a su no eficacia y efectividad. (12)

- A partir de DIRESA y DISA se crean las Direcciones de Redes Integradas de Salud. Se da inicio a la formación de RIS piloto en varias DIRIS.

- Se implementarían varias iniciativas en Infoestructura (REFCON, E-qhali, sistema de citas, historias clínicas electrónicas en hospitales).

Financiamiento de varias zonas piloto a nivel nacional proyecto de apoyo al desarrollo de las Redes Integrales de Salud como parte del fortalecimiento del Sistema Nacional de Salud en el Perú.

Elaboración propia en base a la bibliografía mencionada

funcional, de servicios y clínica (10). Este proceso de integración debe ser visto como un continuo en permanente mejoramiento, entre los tipos y al interior de cada uno de los mismos (tabla 2) (11).
En la figura 1 se incluyen los componentes que deben ser considerados en la conformación de las Redes Integradas de Salud (RIS). Es prioritario que se cuente con una definición de los principios y valores, 
Tabla 2. Tipos de Integración.

\begin{tabular}{ll}
\hline Tipo & Definición \\
\hline \multirow{2}{*}{ Organizacional } & $\begin{array}{l}\text { Integración de organizaciones se une formalmente mediante fusiones o mediante 'colectivos' y/o } \\
\text { virtualmente a través de redes coordinadas de proveedores o por contactos entre organizaciones } \\
\text { negociadas por el comprador }\end{array}$ \\
Funcional & $\begin{array}{l}\text { Integración de soporte no clínico y funciones de back-office, como registros electrónicos de } \\
\text { pacientes. }\end{array}$ \\
De Servicios & $\begin{array}{l}\text { Integración de diferentes servicios clínicos a nivel organizacional, como a través de equipos de } \\
\text { profesionales multidisciplinarios. }\end{array}$ \\
Clínica & $\begin{array}{l}\text { Integración de la atención brindada por profesionales y proveedores a los pacientes en un proceso } \\
\text { único o coherente dentro y / o entre profesiones, como mediante el uso de pautas y protocolos } \\
\text { compartidos }\end{array}$ \\
\hline
\end{tabular}

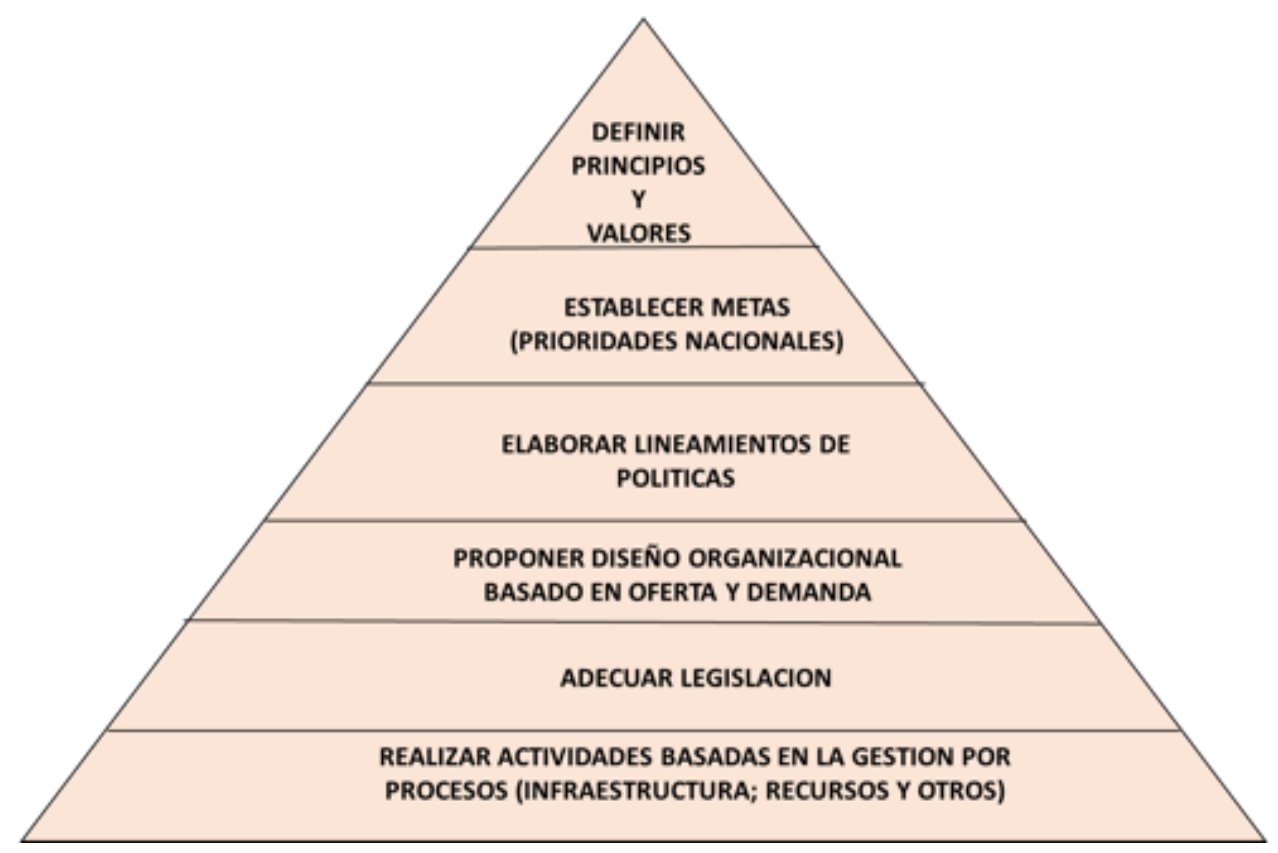

Figura 1. Componentes de una Red Integrada de Salud.

idealmente consensuados con los actores sociales claves, que deben regir el funcionamiento de las RIS. Simultáneamente, se deben establecer las metas nacionales y regionales-, en función a las prioridades nacionales. En función a ello, se deberán elaborar los lineamientos de políticas, entendidas como health policies.

\section{Definición de principios y valores}

Los valores pueden definirse como creencias, principios o estándares de comportamiento significativos, que se refieren a objetivos deseables que motivan la acción (12). Los valores se consideran esenciales para aumentar el compromiso del personal para brindar mejor calidad en los servicios a los clientes en experiencias de éxito en atención integrada
(13). Los valores compartidos entre profesionales y organizaciones se consideran factores importantes en los procesos informales de coordinación y colaboración. Además, una mejor comprensión de los valores de la atención integrada es necesaria para la entrega de una mejor calidad de atención y experiencias de la persona (14).

Se reconoce la limitada información sobre los valores relevantes reales en la atención integrada y su definición. La Organización Mundial de la Salud (OMS) publicó recientemente un informe estratégico que aborda los valores: "Estrategia global sobre servicios de salud integrados y centrados en las personas" (informe provisional) (9). En este informe se afirma que los diferentes enfoques en la prestación de servicios de salud integrados y centrados en las 


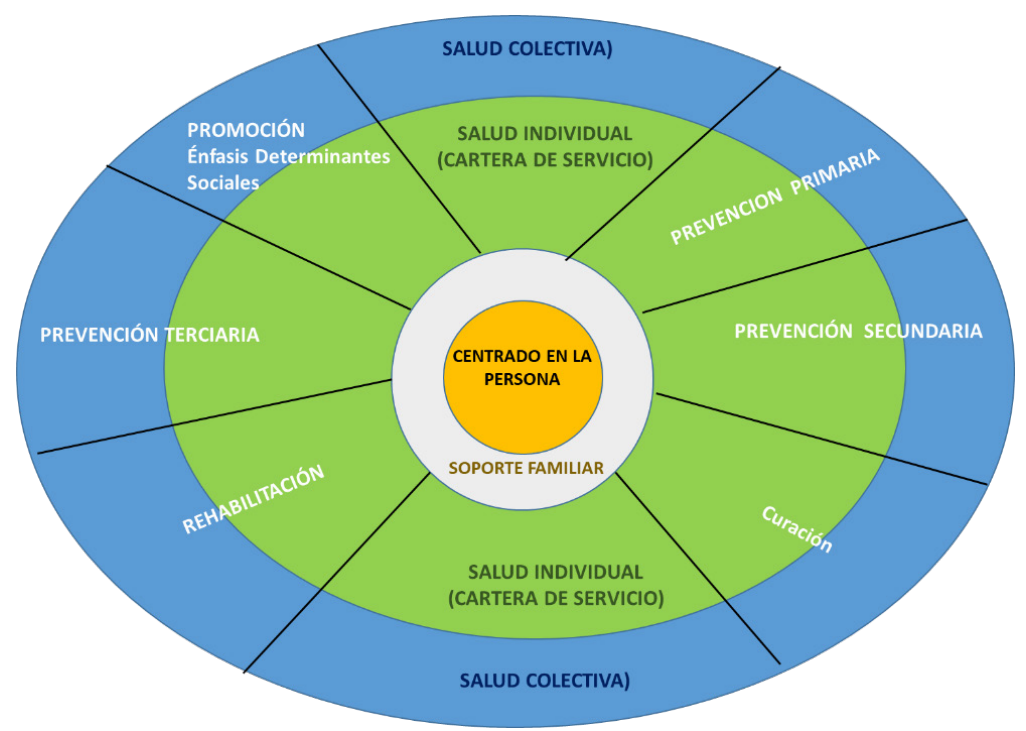

Figura 2. Articulación intra y extramural en las Redes Integradas de Salud.

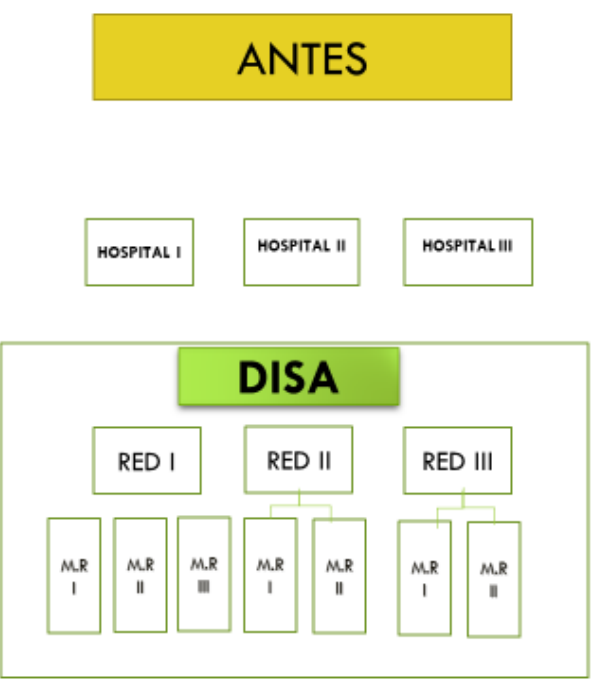

ACTUAL

Figura 3. Nivel estructural de la RIS antes y propuesta actual.

personas "deben basarse en un conjunto común de principios. Estos proporcionan un marco de valores unificadores" (9); este informe contiene un primer conjunto de principios rectores de la atención integrada, desarrollado en colaboración con la Fundación Internacional para la Atención Integrada (8), este conjunto de principios se desarrolló considerando las opiniones de los socios involucrados en el desarrollo del informe provisional, pero los hallazgos aún no se han evaluado sistemáticamente.

Recientemente, Ferrer y Goodwin (15), como parte del trabajo de la International Federation of Integrated Care (IFIC) han desarrollado, con la OMS y expertos internacionales, un conjunto de valores rectores centrales para que las futuras reformas de los sistemas de salud se basen en un conjunto común de objetivos y aspiraciones, se identificaron 16 valores básicos. En el 2018, Zonneveld et al., realizaron una revisión sistemática donde identifican 23 valores reportados en iniciativas de atención integral, que incluye 15 de los 16 principios básicos, el único principio no incluido fue Dotado de derechos y responsabilidades: que todos los ciudadanos deben esperar, ejercer y respetar (16). Por otro lado, Cummings Graduate Institute for Behavioral Health Studies establece ocho valores rectores del cuidado integral (17). 
En este marco de ideas, la propuesta de desarrollo del sistema de salud a través de las RIS, requiere que la Gestión defina los valores que la guiarán, los cuales deben guardar concordancia y complementariedad con los principios y valores establecidos para el sector salud, que aglutinen los esfuerzos hacia políticas de Estado en Salud.

\section{Legislación y prioridades}

La Ley 30885, ley que establece la conformación y el funcionamiento de las Redes Integradas de Salud (RIS) y las funciones: proveer servicios de salud integrales a la población de su ámbito, mediante intervenciones de salud individual y salud pública y abordaje de los determinantes sociales de la salud (1). Por otro lado, se cuenta con la RM 467 que aprueba el Manual de Operaciones de la Direcciones de las Redes Integradas en Salud sólo señala que tienen como misión garantizar la atención oportuna y adecuada de las prestaciones de servicios de salud a los usuarios, así como desarrollar acciones en materia de salud pública e intervenciones sanitarias, emergencia y desastres, docencia e investigación, fortaleciendo la capacidad resolutiva, en el ámbito de su competencia (18).

También se requiere revisar y adecuar la legislación nacional para optimizar el uso de recursos financieros que permita contar con infraestructura, equipamiento e infoestructura adecuada para la atención integrada. Contar con el recurso humano calificado y especializado necesario, acorde a la demanda, evitando la doble percepción; así como poder contar con la activa participación de la sociedad civil, para implementar acciones y denunciar irregularidades.

La aproximación de las RIS guarda cierta concordancia con las definiciones de atención integrada de Leutz (19) y de OMS sobre cuidado integrada (o prestación de servicios de salud integrados) que la define como "Un enfoque para fortalecer los sistemas de salud centrados en las personas a través de la promoción y la prestación integral de servicios de calidad a lo largo de la vida, diseñado de acuerdo con las necesidades multidimensionales de la población y el individuo y entregado por un equipo coordinado multidisciplinario de proveedores que trabajan en diferentes entornos y niveles de atención. Debe gestionarse eficazmente para garantizar resultados óptimos y el uso adecuado de los recursos en función de la mejor evidencia disponible, con bucles de retroalimentación para mejorar continuamente el rendimiento y abordar las causas ascendentes de mala salud y promover el bienestar a través de acciones intersectoriales y multisectoriales" (20).

En el marco de intervenciones en salud individual y salud pública/salud colectiva, éstas se deben desarrollar (implementar, monitorear y evaluar) cambiando el centro de atención, priorizando la atención integral e integrada que se enfoque en el cuidado de la salud y promoción del bienestar, se centre en la persona-familia-comunidad y tenga una aproximación territorial. Ello incluye mejorar las estrategias e intervenciones en los problemas sanitarios prioritarios desde los determinantes sociales (agua y saneamiento, control vectorial), prevención social (salud materno-infantil, enfermedades crónicas no transmisibles); así mismo, se debe garantizar la atención individual de la población afectada por problemas de salud, contando con la participación de profesionales de salud con las competencias necesarias (especialización o entrenamiento) para los problemas sanitarios priorizados.

\section{Necesidad de un modelo}

El modelo de atención integral de salud basado en familia y comunidad (MAIS-BFC), aún vigente, se desarrolló con énfasis en las actividades preventivopromocionales en el primer nivel de atención, promoviendo la identificación de factores de riesgo en el ámbito comunitario y designando paquetes de intervenciones para cada etapa de vida $(21,22)$. La OMS plantea pasar a un modelo de cuidados por curso de vida, que considere la salud como un proceso dinámico que considere intervenciones más allá de la atención específica, que considere que la salud de un individuo es influenciada por sus antecedentes con influencia en su salud en el futuro (23). Puertas et al., basados en la experiencia de Honduras señalan que la organización en RIS, plantea intervenciones con un diseño de soluciones basado en la perspectiva y experiencias de las personas, y su preferencia con la finalidad de fortalecer el cuidado de las mismas y tomar decisiones compartidas con la familia y la comunidad (24).

No se conoce desarrollo alguno de una propuesta diferente del modelo de atención para las RIS. Por tanto, se requiere ampliar y actualizar el modelo. Contextualizar un modelo de cuidado para la atención en las RIS que tenga la flexibilidad para adecuarse a las diversas realidades socio-económicas y culturales del país; así como las características de la salud de la comunidad, de las familias y cada persona por etapa de vida. 
El modelo debe abordar los problemas de salud pública incluidos sus determinantes sociales, y problemas de salud individual (desde la promoción de la salud prevención primaria -de la enfermedad- hasta la prevención terciaria -de las secuelas-). Ello implica que se deben desarrollar actividades de promoción y prevención primaria, secundaria y terciaria a nivel individual y de la comunidad, entendida como una población geográficamente determinada o como la familia como el núcleo más pequeño. Por ejemplo, se pueden realizar actividades de promoción sobre alimentación saludable o actividad física a un grupo poblacional general o específico a nivel extramural, o se puede hacer la misma actividad promocional a un paciente o grupo de pacientes a nivel intramural (con o sin enfermedad). Puedo realizar una actividad de prevención primaria, como dosaje de creatinina, a población en riesgo para identificación temprana de pacientes con insuficiencia renal en el espacio extramural; o puedo realizar la misma actividad sobre una persona con los mismos criterios de riesgo que acude a un establecimiento de salud (actividad intramural). También puedo realizar acciones de prevención secundaria acompañadas de actividades educativas para prevenir las complicaciones (por ejemplo, en pacientes diabéticos sin complicaciones), tanto a nivel extramural como a nivel intramuros para los pacientes y sus familiares, quienes son el soporte para garantizar una buena evolución del paciente. Finalmente, lo mismo ocurre cuando deseamos realizar acciones de prevención terciaria acompañadas de actividades educativas para prevenir las secuelas (por ejemplo, paciente con retinopatía diabética), a nivel extramural o intramural (figura 2).

\section{Mirando las RIS desde la población}

En muchos países, como el nuestro, la transición epidemiológica con persistencia de enfermedades transmisibles y aparición de las enfermedades crónicas, se ha expresado en el envejecimiento poblacional y el aumento de la carga de enfermedades crónicas. Se sabe que ambas condiciones aumentan los costos de atención médica y la utilización de muchos países (25), y que en el largo plazo genera dificultades para satisfacer las crecientes demandas de atención. Ello va acompañado del efecto acumulativo de cambios sociodemográficos, económicos y ambientales, así como las crecientes expectativas de atención, en especial en el ámbito urbano. Estas nuevas demandas conllevan a brindar atención que sea proactiva, integral y continua, y eso es construido sobre una relación sostenible entre el paciente y el proveedor (26).
En concordancia con lo desarrollado en el cuidado integral, se debe resaltar la importancia de la atención basada en lugar y centrada en la persona, lo cual debe brindar resultados relevantes para las personas. La atención centrada en la persona debe expresar una integración exitosa de buenas prácticas en materia de salud, atención y bienestar. Ello requiere un mayor énfasis en las acciones de control de gestión (supervisión, monitoreo y evaluación). Se espera que un enfoque centrado en la persona se convierta en una práctica estándar. La atención basada en lugar debe permitir prevenir pobre salud y reducir las inequidades en salud, como elemento central en cuidado integral, así como atender emergencias y desastres, tanto para mejorar la vida de las personas como para reducir la demanda de servicios de salud y atención. Este cambio en el foco de atención permitirá lograr con éxito la completa transformación del sistema.

Para que la transformación se pueda dar, se requiere brindar atención enfocada en el logro de resultados y centrada en la persona-familia-comunidad con énfasis en el autocuidado y participación de la comunidad, para poder llevar una vida más saludable, más independiente y plena. Asimismo, un enfoque de salud de la población, debe prevenir la pobre salud y reducir los riesgos y las inequidades a través de movilizar todos los activos de los actores sociales para ayudar a las personas a llevar vidas más saludables y felices con menos necesidad y vulnerabilidad. El énfasis en el autocuidado, requiere un fuerte énfasis en la promoción de la salud en las edades tempranas de la vida lo cual debe ayudar a mejorar la cultura de salud en la población y crear mayor conciencia de la responsabilidad individual y social por el autocuidado. Finalmente, se deben aprender de los aciertos y errores de la participación comunitaria en la gestión de los establecimientos de salud, como ocurrió con los CLAS.

\section{Mirando RIS desde la oferta}

El sistema de salud peruano es considerado un sistema segmentado y fragmentado. La fragmentación existente es el resultado de la sobre-medicalización, subespecialización excesiva y un modelo de atención curativa vertical orientado a la enfermedad por separado. Tales enfoques fragmentados reducen la capacidad del sistema de salud para proporcionar continuidad de la atención, lo que conduce a dificultades en el acceso oportuno a la atención, la prestación de servicios de baja calidad, la duplicación de esfuerzos y el uso ineficiente de los recursos. 
Estos enfoques también crean una baja satisfacción del usuario del servicio y brechas en la atención de pacientes con multimorbilidades (27).

Diversos gobiernos han implementado reformas estructurales y financieras para alejarse de los modelos de atención fragmentados centrados en el proveedor y reorientarlos según los principios de integración para garantizar que todos tengan acceso a una atención continua que sea receptiva, coordinada y en línea con las necesidades de las personas en todo momento. La integración exitosa también asegura que los servicios de atención médica a lo largo de este continuo de atención sean de calidad aceptable, es decir, eficaces, seguros y centrados en las personas (28).

Los servicios de salud integrados, basados en sólidas funciones de atención primaria y salud pública, contribuyen directamente a una mejor condición de salud, bienestar y calidad de vida: ello debe generar beneficios económicos, sociales e individuales. $\mathrm{La}$ atención integrada favorece a un mejor acceso a los servicios, menos hospitalizaciones innecesarias y reingresos, mejor adherencia al tratamiento, mayor satisfacción del paciente, alfabetización en salud y autocuidado, mayor satisfacción laboral para los trabajadores de la salud y mejores resultados generales de salud (29-32). También se evidencia efectividad de los servicios de salud integrados, particularmente para el manejo de enfermedades no transmisibles y enfermedades crónicas (33). Sin embargo, no hay evidencia concluyente del costo-efectividad para intervenciones complejas, lo cual hasta ahora no es concluyente (34), y la evidencia clara de la efectividad de cambios diversos y complejos es difícil de evidenciar debido a limitaciones metodológicas en definición, medición y evaluación de la atención integrada.

Los servicios de salud integrados deben mejorar la equLos servicios de salud integrados deben mejorar la equidad; fomentar servicios basados en las necesidades holísticas de la población y brindar diferentes tipos de atención a lo largo de la vida, desde la protección y promoción de la salud hasta la atención a largo plazo, rehabilitación y cuidados paliativos. Así como una respuesta adecuada en emergencias y desastres. Este continuo de atención se debe coordinar a través de los diferentes niveles de atención dentro y fuera del sector de la salud (35); este enfoque integrado para la prestación de servicios es crucial para lograr una eficiente cobertura universal de salud (36).

Al interior del sector salud implica integrar la atención primaria y la salud pública, y los niveles de atención. La salud pública proporciona los objetivos generales para mejorar la salud, de forma que los servicios deben diseñarse y organizarse en torno a las prioridades de salud pública. La evidencia muestra los siguientes cambios operativos para apoyar esta integración: (a) adaptar las acciones y recursos de mejora de la salud para llegar a las áreas y grupos sociales más desfavorecidos; (b) crear capacidad en atención primaria para brindar promoción proactiva y atención preventiva; (c) trabajar en prácticas modernas de medicina familiar, desde la promoción y prevención de la salud hasta la rehabilitación y cuidados paliativos; (d) desarrollar intervenciones tempranas para evitar el escalamiento de las necesidades sociales y de salud; y (e) enmarcar la atención a las personas dentro de una perspectiva de población más amplia para mejorar la equidad en salud y la cohesión social (37). Esta integración debe conseguir resultados de mediano largo plazo respondiendo a estilos de vida poco saludables y factores de riesgo ambientales y a abordar otros factores de riesgo y determinantes de la salud.

La integración de la atención primaria, secundaria y terciaria debe responder al aumento de las enfermedades crónicas y multimorbilidad, y abordar la fragmentación de la atención brindada por múltiples proveedores, en muchos entornos y en muchos niveles. Esta vía se centra en integrar la prestación de servicios entre proveedores y los tipos de atención y entornos. Incluye la intersección de la atención primaria con el hospital y otros tipos de atención institucionalizada; así como con la guardería y los servicios de enfermería diaria en el hogar (38). La integración de hospitales con establecimientos de primer nivel es un vínculo muy importante para garantizar una atención continua para los pacientes. En muchos países se están realizando transformaciones significativas creando condiciones para que los hospitales se integren en una red de prestación de servicios viéndose a sí mismos como organizaciones flexibles que reúnen recursos escasos y funcionan para el bien público y que logre la continuidad y la atención centrada en las personas (39).

Lo más importante es mejorar los resultados y equidad en salud; ello requiere un enfoque innovador para abordar los determinantes sociales que afectan la salud de la población y considerar a la salud en todas las políticas de estado, con el apoyo de un compromiso político y liderazgo fuerte $(40,41)$. La acción y políticas multisectoriales requieren integrar a todos los sectores para abordar los determinantes sociales, culturales, ambientales, políticos y comerciales de 
la salud y bienestar. Ello implica reposicionar al sector salud dentro del ámbito del desarrollo. Las acciones y alianzas intersectoriales para la salud y bienestar mejoran la planificación, coordinación y prestación de servicios de diferentes sectores. Las políticas integradas que involucran a muchos sectores aseguran la protección social y la cohesión general en la sociedad, y maximizan la equidad en los resultados de salud (42). Además, para hacer frente a la discapacidad, envejecimiento y condiciones crónicas, se debe fortalecer la integración de los programas sociales, servicios sociales y servicios de salud. Las estrategias para hacer esto incluyen la participación de los trabajadores sociales, la planificación conjunta, la compra y la prestación de servicios, la agrupación de fondos y la implementación de mecanismos de coordinación y gobernanza. El trabajo conjunto puede ayudar a lograr los objetivos sociales y de salud compartidos de proporcionar atención a largo plazo en el hogar y la comunidad.

En base a lo desarrollado haremos una propuesta sobre la organización de las Redes integradas de salud en las grandes ciudades. Servicios de salud integrados en todas las etapas del ciclo de vida, que se centren en las prioridades sanitarias del territorio, ello implica trascender del "enfoque materno-infantil" para pasar al desarrollo integral de la mujer, al desarrollo infantil temprano, adolescencia, adultez y al envejecimiento con dignidad.

\section{Diseño de las redes integradas de salud (RIS) en las grandes ciudades}

La realidad sanitaria es heterogénea al interior del país, desde una mirada de la oferta y de la demanda. Para pensar en las RIS debemos abordar apropiadamente la realidad sanitaria actual existente en las grandes ciudades y desarrollar iniciativas para evitar el escalamiento de los problemas sanitarios en las personas y en la población (sociedad). Ello implica considerarla como una política de Estado en salud, de concepción integral e integrada que contenga el desarrollo de un trabajo intramural y extramural. Pensar nuestra función sanitaria implica también abordar los temas organizacionales (meso) y los temas operativos (micro), basándose en los siguientes principios: Basado en el paciente, integralidad de la atención, calidad de atención, oportunidad y continuidad de atención.

A nivel intramural, se evidencia un exceso de demanda de atención en los hospitales con una limitada o casi nula contrarreferencia de los mismos a los establecimientos de primer nivel. Este es un problema cultural con comportamientos inapropiados de parte de los prestadores y de los pacientes. Para el adecuado funcionamiento de la RIS, la lógica de la prestación debe cambiar, más allá del énfasis en la atención materno-infantil, que no debe ser descuidada, debe tener un enfoque integral basado en el nuevo perfil epidemiológico, que desarrolle a modo de motor impulsor de la RIS, un robusto sistema de Referencia y contrarreferencia integrado (emergencias, ambulatorio, hospitalización).

Como la función modela la estructura, la propuesta implica adecuar la actual infraestructura, infoestructura, recurso humano, equipamiento y los procesos operativos (operaciones) al interior de los establecimientos de la RIS; varios de ellos han sido adecuados para la atención centrada en el componente materno infantil. Por tanto, se requiere contar con establecimientos de salud certificados y adecuados a esta nueva propuesta.

Por ello, se considera que se deben brindar atención de especialidades médicas de alta demanda. Se debe aspirar a que cada RIS cuente con la capacidad resolutiva para atender las necesidades de salud de la población en el primer nivel, descongestionando los hospitales, ya saturados. Los establecimientos de área (popularmente llamados "cabeceras de las RIS") o nivel I-4 inmediato inferior a los Hospitales, deberán no sólo brindar atención de 24 horas (gíneco-obstetricia y pediatría), sino brindar la atención con especialistas en cardiología, medicina interna, reumatología, endocrinología, oftalmología, otorrinolaringología y medicina física y rehabilitación, atención de cirugía menor y electiva de las patologías que requieran de especialistas en cirugía general y anestesiología; complementando con la atención en odontología, nutrición y psicología, laboratorio clínico e imagenología (rayos X y ecografía). Por ejemplo, debe existir servicios de consulta externa de cardiología, mañana y tarde, con el equipamiento apropiado (holter, MAPA, ecocardiograma, electrocardiógrafo y equipo de paro). Además, al interior de cada RIS, se deberán considerar un pequeño número de instituciones prestadoras de servicios de salud (IPRESS) que también brinden atención 24 horas, con énfasis en la población materno-infantil y en la población vulnerable (personas con enfermedades crónicas y personas adultas mayores (medicina familiar y comunitaria). En la figura 3 se muestra la arquitectura sugerida. 
Además, a nivel de la Dirección de redes integradas de Salud (DIRIS), es recomendable evaluar la posibilidad de centralizar, en cada una de las RIS (léase, establecimientos de salud I-4), servicios de imagenología, laboratorio clínico y, medicina física y rehabilitación, entre otros, a fin de optimizar el uso de equipamiento y otros recursos (incluyendo insumos, mantenimiento, recursos humanos y recursos financieros), y disminuir los riesgos de la prestación. Para ello, existen diversas posibilidades que deben ser analizados a la luz de la experiencia y data de estudios de evaluación económica. Estos escenarios incluyen: Compra de equipos, comodato de equipos o servicios tercerizados.

Por otro lado, a nivel de la actividad de salud pública (intra y extramural) se requiere abordar acciones que tengan que ver con los determinantes sociales y las responsabilidades personales de cuidado de la salud individual, familiar y comunitaria; lo que se señala, según el modelo conceptual de Valentin y col. (25), como el cuidado basado en población en los tres niveles (macro, meso y micro). Diversas actividades en esta área tienen que ver con actividades de promoción de estilos de vida saludables y prevención primaria; acciones de control de saneamiento básico, alimentación saludable, entre otras. Asimismo, el Estado debe desarrollar acciones específicas, para todas las etapas de vida, a través de programas nacionales específicos o de actividades extramurales, de manera articulada y coordinada con la sociedad civil (organizaciones sociales de base) y el gobierno local, responsable de la Gestión de Atención Primaria de Salud (Ley Orgánica de Municipalidades, artículo 80) y cuyo ámbito de aplicación comprende las categorías I-1 al I-4 (Resolución Ministerial 12042006-SA).

Asimismo, se requiere contar en la DIRIS con una Unidad Funcional de Control de Calidad en Salud Pública (UFCCP); por ejemplo, en el caso de Tuberculosis (baciloscopía, cultivo y pruebas de sensibilidad diagnóstica) y en VIH (confirmación diagnóstica, CD4 y carga viral) y metrología (calidad de mediciones laboratoriales). Además, contar con una Unidad Funcional de Control de Calidad en Salud Individual (UFCCI), que evalúe: (a) Asistencia de profesionales y programación trimestral; (b) Stock de medicamentos según capacidad resolutiva; (c) Mantenimiento de equipos; (d) Limpieza de las IPRESS, (e) Calidad de atención en pacientes SIS, mediante el adecuado llenado del formato único de atención (FUA), historias clínicas, exámenes auxiliares y recetas; (f) análisis de muertes maternas y muertes neonatales; Categorización y Acreditación (g) análisis de índices entomológicos; (h) análisis de adherencia de guías de práctica clínica; (i) análisis de cumplimiento de las referencias y contrarreferencias, entre otros.

El trabajo intramural y extramural, se debe expresar en el desarrollo de alianzas y coordinaciones con la comunidad de manera intensiva, repotenciando la credibilidad del sector salud (salud y sociedad), aumentando la extensión de uso de los establecimientos de salud e identificando a la población con mayor vulnerabilidad, que permitan contar con la participación de la comunidad organizada en la cogestión de los servicios. Asimismo, se requiere un trabajo integrado con otros actores o prestadores que permita revertir la segmentación y fragmentación del sistema vigente.

El nuevo enfoque implica un trabajo sectorial en la adecuación del recurso humano a este modelo que permita contar con el recurso humano potenciado en cantidad, calidad y especialidad para ambas actividades y el equipamiento actualizado a esta nueva prestación.

También se requiere contar con los desarrollos adecuados de infoestructura, la cual debe ir acompañada de una capacitación permanente del recurso humano (especialmente de quienes no son nativos digitales). Se requiere dotar a los establecimientos de salud con el adecuado ancho de banda que garantice su funcionamiento, con los equipos informáticos, aplicativos y software para la historia clínica electrónica, programación de citas, referencia y contrarreferencia, telesalud, con las correspondientes interfaces que faciliten la interoperabilidad de los programas.

Finalmente, y no por ello menos importante, se requiere impulsar un significativo cambio cultural, una cultura de calidad y servicio, donde las distintas actividades sean desarrolladas como procesos operativos detallados. Esto es, pensar en que todas las actividades en los establecimientos de salud, RIS y DIRIS sean realizadas a través de procedimientos operativos estandarizados, los cuales deben contar con las aprobaciones administrativas correspondientes.

Si se logra que las redes de servicios de salud sean dinámicas en su desarrollo y generen realmente servicios acordes a la salud de la población en las grandes ciudades, las redes habrán logrado la sinergia que genere salud diariamente. 


\section{Declaración de financiamiento y de conflictos de interés:}

El estudio contó con el apoyo y autorización para publicación de la DIRIS Lima Norte, obteniéndose información a partir de la revisión de literatura y desarrollo de la propuesta de implementación de Redes Integradas de Salud (RIS). Los autores declaran no tener conflictos de interés.

\section{Correspondencia:}

Luis Fernando Llanos Zavalaga

Dirección: Calle Las Lilas 435 - Urb. San Eugenio. Lince.

Teléfono: (511)-999660680

Correo electrónico: luis.llanos@upch.pe

\section{REFERENCIAS BIBLIOGRÁFICAS}

1. Congreso de la Republica. Ley $\mathrm{N}^{\mathrm{o}} 30885$. Ley que establece la conformación y el funcionamiento de las redes integradas de salud (RIS). Lima: Diario Oficial El Peruano; 18 de diciembre del 2018. (Citado el 22 de noviembre del 2019) Disponible en: https://busquedas.elperuano.pe/normaslegales/leyque-establece-la-conformacion-y-el-funcionamientode-las-ley-n-30885-1724734-2/

2. Bermejo R. Desarrollo de los Servicios de Salud. OPS/OMS. Pro Salute Novi Mundi. Historia de la Organización Panamericana de la Salud. Cien años de Cooperación al Perú. Washington DC: Organización Panamericana de la Salud; 1992.

3. Alcalde-Rabanal JE, Lazo-Gonzales O, Nigenda G. Sistema de salud de Perú. Salud Pública Mex. 2011; 53(supl 2):S243-S254.

4. Sánchez-Moreno, F. El sistema nacional de salud en el Perú. Rev Peru Med Exp Salud Publica. 2014; 31(4):747-53.

5. Lazo-Gonzales O, Alcalde-Rabanal J, EspinosaHenao O. El sistema de Salud en Perú situación y desafíos. Lima: Colegio Médico del Perú; 2016.

6. Centrángolo O, Bertranou F, Casanova 1, Casali P. El Sistema de Salud del Perú: situación actual y estrategias para orientar la extensión de la cobertura contributiva. Lima Perú: EsSALUD-OIT; 2013.

7. Cosavalente O, Zevallos L, Fasanando J, Cuba S. Proceso de transformación hacia las Redes Integradas de Salud en el Perú. Rev Per Medicina Experimental y Salud Pública. 2019; 36(2):319-325.

8. World Health Organization. Primary health care systems (PRIMASYS): case study from Peru, abridged version. Geneva: World Health Organization; 2017.

9. Organización Panamericana de la Salud (OPS/OMS).
Sistemas de Salud basados en la Atención Primaria de Salud - Estrategias para el Desarrollo de los equipos de APS. Serie La Renovación de la Atención Primaria de Salud en las Américas. 2008; 1:13.

10. Lewis R, Rosen R, Goodwin N, Dixon J. Where next for integrated care organizations in the English NHS? London: The King's Fund; 2010. (Citado el 22 de noviembre del 2019) Disponible en: https:// www.nuffieldtrust.org.uk/files/2017-01/where-nextintegrated-care-english-nhs-web-final.pdf

11. World Health Organization Regional Office for Europe. Integrated care models: an overview Working document October 2016. Geneva: World Health Organization;2016

12. Schwartz, SH. An overview of the Schwartz theory of basic values. Online readings in Psychology and Culture. 2012; 2:11. DOI: https:// doi. org/10.9707/2307-0919.1116

13. Goodwin N. Taking integrated care forward: the need for shared values. Int J Integr Care. 2013; 13(2):0-0. DOI: http://doi.org/10.5334/ijic.1180

14. Minkman MMN. Values and Principles of Integrated Care. Int J Integr Care, 2016; 16(1):2. DOI: https:// doi.org/10.5334/ ijic. 2458

15. Ferrer L, Goodwin N. What are the principles that underpin integrated care? Int J Integr Care. 2014; 14:e037. doi: $10.5334 / \mathrm{ijic} .1884$

16. Zonneveld N, Driessen N, Stüssgen RAJ, Minkman MMN. Values of integrated care: A systematic review. Int J Integr Care. 2018; 18(4):1-12. DOI: https://doi. org/10.5334/ijic. 4172

17. Cummings Graduate Institute for Behavioral Health Studies. Mission, Vision, and Values Phoenix, Arizona: Cummings Graduate Institute for Behavioral Health Studies; 2017. (Citado 22 de noviembre del 2019) Disponible en: https://cummingsinstitute.com/ mission-vision-values/

18. Ministerio de Salud. Resolución Ministerial $\mathrm{N}^{\circ}$ 467-2017-MINSA. Manual de Operaciones de las Redes Integradas de Salud de Lima Metropolitana. Lima: Ministerio de Salud; 2017. (Citado el 22 de noviembre del 2019) Disponible en: https://www. minsa.gob.pe/transparencia/index.asp?op=117

19. Leutz WN. Five Laws for Integrating Medical and Social Services: Lessons from the United States and the United Kingdom. Milbank Q. 1999; 77(1):77110. doi: 10.1111/1468-0009.00125

20. World Health Organization Regional Office for Europe. Strengthening people-centred health systems in the WHO European Region: Framework for action on integrated health services delivery. Geneva: World Health Organization Regional Office for Europe; 2016. (Citado el 22 de noviembre del 2019) Disponible en: http://www.euro.who.int/_data/ assets/pdf file/0004/315787/66wd15e FFA IHSD_160535.pdf?ua $=1$ 
21. Medicus Mundi Navarra, Salud Sin Límites Perú. Aportes para la operativización del modelo de atención integral de salud basado en familia y comunidad en el primer nivel. Lima: Salud Sin Límites Perú; 2012. (Citado el 22 de noviembre del 2019). Disponible en: http://bvs.minsa.gob.pe/local/ MINSA/1880.pdf

22. Cuba-Fuentes M, Romero-Albino Z, Dominguez R, Rojas L, Villanueva R. Dimensiones claves para fortalecer la atención primaria en el Perú a cuarenta años de Alma Ata. An Fac med. 2018:79(4):346-50. doi: http://dx.doi. org/10.15381/anales.v79i4.15642

23. Organización Panamericana de la Salud. Construyendo la salud en el curso de vida. Washington, D.C:OPS; 2018.

24. Puertas EB, Martínez RA, Figueroa GS, Hidalgo F. Integración de redes de servicios de salud en Honduras: valoración comparativa del planteamiento teórico y de la aplicación práctica en cinco redes del país. Rev Panam Salud Pública. 2018; 42:135. doi: https://doi.org/10.26633/RPSP.2018.135

25. Valentijn PP, Schepman SM, Opheij W, Bruijnzeels MA. Understanding integrated care: A comprehensive conceptual framework based on the integrative functions of primary care. Int J Integr Care. 2013; 13: 010 .

26. WHO. World Health Report 2008: primary health care (now more than ever); WHO. Everybody's business: strengthening health systems to improve health outcomes 2007. Geneva: World Health Organization; 2007

27. World Health Organization. WHO-UNICEF vision for primary health care in the 21st century in support of the Global Conference on Primary Health Care on 25-26 October 2018. Astana, Kazakhstan: World Health Organization; 2018.

28. World Health Organization. Technical series on primary health care: Integrating Health services. Geneva: World Health Organization; 2018.

29. Nolte E, Pitchforth E. What is the evidence on the economic impacts of integrated care? Policy summary 11. Copenhagen: WHO Regional Office for Europe on behalf of the European Observatory on Health Systems and Policies; 2014. (Citado el 22 de noviembre del 2019) Disponible en: http://www. euro.who.int/_data/ assets/pdf_file/0019/251434/ What-is-the-evidence-on-the-economic-impacts-ofintegrated-care.pdf

30. Nolte E, McKee M. Caring for people with chronic conditions. A health system perspective. Copenhagen: WHO Regional Office for Europe on behalf of the European Observatory on Health Systems and Policies; 2008.
31. McDonald KM, Sundaram V, Bravata DM, et al. Closing the Quality Gap: a Critical Analysis of Quality Improvement Strategies. Vol. 7: Care Coordination. Rockville: Agency for Healthcare Research and Quality (US); 2007.

32. Dudley L, Garner P. Strategies for integrating primary health services in low- and middle-income countries at the point of delivery. Cochrane Database Syst Rev. 2011;7: CD003318. doi: 10.1002/14651858. CD003318.pub3

33. World Health Organization. People-centred and integrated health services: an overview of the evidence. Geneva: World Health Organization; 2015.

34. Nolte E, Pitchforth E. What is the evidence on the economic impacts of integrated care? Policy summary 11. Copenhagen: WHO Regional Office for Europe on behalf of the European Observatory on Health Systems and Policies; 2014.

35. Kodner DL, Spreeuwenberg C. Integrated care: meaning, logic, applications, and implications - a discussion paper. Int J Integr Care. 2002; 2:e12.

36. World Health Organization. Technical series on primary health care. Integrating Health services. Geneva: World Health Organization; 2018.

37. Rawaf S. Policy Brief: Closing the gap between public health and primary health care through integration: Models of integration to improve health and achieve universal health coverage. Geneva: World Health Organization; 2018.

38. World Health Organization. Strengthening peoplecentred health systems in the WHO European Region: framework for action on integrated health services delivery. Copenhagen: World Health Organization Regional Office for Europe; 2016.

39. World Health Organization. A global vision for person-and community centred hospitals in a primary health care-based health system. Geneva: World Health Organization; 2018.

40. World Health Organization. The World Health Report 2008: primary health care (now more than ever). Geneva: World Health Organization; 2008.

41. Jakab M, Farrington J, Borgermans L, Mantingh F, editors. Health systems respond to noncommunicable diseases: time for ambition. Copenhagen: WHO Regional Office for Europe; 2018.

42. World Health Organization Regional Office for Europe. Strengthening people-centred health systems in the WHO European Region: framework for action on integrated health services delivery: Working document: Regional Committee for Europe 66th session. Copenhagen, Denmark: World Health Organization Regional Office for Europe; 2016.

Recibido: $18 / 11 / 2019$

Aceptado: 27/12/2019 